\title{
BMJ Open Detection of intracranial hypertension in children using optical coherence tomography: a systematic review
}

\author{
Sohaib R Rufai (i) , ${ }^{1,2,3}$ Michael Hisaund, ${ }^{1}$ Noor ul Owase Jeelani, ${ }^{2}$ \\ Rebecca J McLean ${ }^{1}$
}

To cite: Rufai SR, Hisaund M, Jeelani $\mathrm{NuO}$, et al. Detection of intracranial hypertension in children using optical coherence tomography: a systematic review. BMJ Open 2021;11:e046935. doi:10.1136/ bmjopen-2020-046935

- Prepublication history and additional supplemental material for this paper are available online. To view these files, please visit the journal online. (http://dx.doi.org/10.1136/ bmjopen-2020-046935).

Received 14 November 2020 Accepted 05 July 2021

Check for updates

(C) Author(s) (or their employer(s)) 2021. Re-use permitted under CC BY. Published by BMJ.

${ }^{1}$ University of Leicester Ulverscroft Eye Unit, Leicester Royal Infirmary, Leicester, UK ${ }^{2} \mathrm{UCL}$ Great Ormond Street Institute of Child Health and Craniofacial Unit, Great Ormond Street Hospital for Children,

London, UK

${ }^{3}$ Clinical and Academic Department of Ophthalmology, Great Ormond Street Hospital for Children, London, UK

Correspondence to Dr Rebecca J McLean; rjm19@leicester.ac.uk

\section{ABSTRACT}

Objectives To evaluate the diagnostic capability of optical coherence tomography (OCT) in children aged under 18 years old with intracranial hypertension $(\mathrm{IH})$.

Design Systematic review.

Methods We conducted a systematic review using the following platforms to search the keywords 'optical coherence tomography' and 'intracranial hypertension' from inception to 2 April 2020: Cochrane Central Register of Controlled Trials, EMBASE, MEDLINE, PubMed and Web of Science, without language restrictions. Our search returned 2729 records, screened by two independent screeners. Studies were graded according to the Oxford Centre for Evidence-Based Medicine and National Institutes of Health Quality Assessment Tool for observational studies.

Results Twenty-one studies were included. Conditions included craniosynostosis ( $\mathrm{n}=354$ patients), idiopathic $\mathrm{IH}(\mathrm{IH} ; \mathrm{n}=102)$, space-occupying lesion (SOL; $\mathrm{n}=42$ ) and other pathology $(n=29)$. OCT measures included optic nerve parameters, rim parameters (notably retinal nerve fibre layer thickness) and retinal parameters. Levels of evidence included $2 b$ ( $n=13$ studies), $3 b(n=4)$ and 4 $(n=4)$. Quality of 10 studies was fair and 11 poor. There was inconsistency in OCT parameters and reference measures studied, although OCT did demonstrate good diagnostic capability for IH in craniosynostosis, IIH and SOL.

Conclusions This systematic review identified various studies involving OCT to assist diagnosis and management of $\mathrm{IH}$ in children with craniosynostosis, IIH, SOL and other pathology, in conjunction with established clinical measures of intracranial pressure. However, no level 1 evidence was identified. Validating prospective studies are, therefore, required to determine optimal OCT parameters in this role and to develop formal clinical guidelines. PROSPERO registration number CRD42019154254.

\section{INTRODUCTION}

Intracranial hypertension (IH) was first described by Quincke in 1896 and remains a subject of major clinical importance. ${ }^{1}$ IH affects between 0.63 and 0.71 per 100 000 children. $^{2}{ }^{3}$ Unaddressed IH can inflict devastating sequelae including visual impairment, neurocognitive delay, disability and death. ${ }^{45}$ Subacute pathology in children can

\section{Strengths and limitations of this study}

- This is the first systematic review to evaluate the role of optical coherence tomography (OCT) in paediatric intracranial hypertension $(\mathrm{IH})$.

- This systematic review was conducted in accordance with rigorous Cochrane methodology.

- A broad search strategy was employed, without date or language restrictions, to identify all relevant evidence.

- There was inconsistency in evidence levels and quality across the included studies, although OCT did demonstrate good diagnostic capability for paediatric $\mathrm{IH}$.

- Validating prospective studies are required to develop formal clinical guidelines for OCT in paediatric $\mathrm{IH}$.

cause insidious IH which may pose deleterious effects on the brain and vision before clinical detection. Thus, prompt detection and timely intervention is key in preventing or limiting the sequelae of IH.

Assessment of intracranial pressure (ICP) in children is notoriously difficult. Direct intraparenchymal measurement represents the gold standard, but carries numerous disadvantages including the need for overnight hospital admission, general anaesthesia and significant risk. ${ }^{6}$ An ideal surveillance method would be highly sensitive, specific, safe, highly reproducible, rapid, noninvasive and child friendly with the capability to record serial measurements. Existing measures fail to fully satisfy all these criteria and often yield equivocal results in young children, including fundus examination, ${ }^{8}$ B-scan ocular ultrasound, ${ }^{9}{ }^{10}$ radiology ${ }^{11}$ and visual evoked potentials. ${ }^{12} 13$

IH causes optic nerve and retinal changes, which can be detected and quantified using optical coherence tomography (OCT) - a non-invasive imaging method to acquire ultrahigh resolution cross-sectional images of the optic nerve and retina within seconds. ${ }^{14}$ 
OCT has been successfully used to study the normal and abnormal development of the optic nerve ${ }^{15}$ and fovea ${ }^{16}$ in children, plus various conditions associated with $\mathrm{IH}^{17-23}$ Here, we conducted a systematic review to assess the role of OCT in detecting IH in children.

\section{METHODS}

This systematic review was conducted in accordance with Preferred Reporting Items for Systematic Reviews and Meta-Analyses (PRISMA) guidelines ${ }^{24}$ and the Cochrane Handbook..$^{25}$ The protocol is registered on PROSPERO ${ }^{26}$ and published in BMJ Open. ${ }^{27}$

\section{Eligibility criteria for considering studies for this review}

Eligibility criteria were established a priori and included OCT studies of children (aged under 18 years) with IH. Level 4 evidence and above was included, as per the Oxford Centre for Evidence-based Medicine (CEBM). ${ }^{28}$ Exclusion criteria were: (1) studies of adults aged 18 or over; (2) studies not pertaining to IH; (3) studies not using OCT and (4) case reports and expert opinion without critical appraisal.

\section{Search methods for identifying studies}

Medical subject headings terms for 'optical coherence tomography' and 'intracranial hypertension' were entered into search platforms: Cochrane Central Register of Controlled Trials, MEDLINE, EMBASE, PubMed and Web of Science. Online supplemental appendix 1 contains full details of our search terms and strategy. EndNote V.X9 (Thomson Reuters, New York, New York, USA) was used to manage data. No date/language restrictions were stipulated.

\section{Study selection}

A three-stage, independent screening process was employed by two screeners (SRR and RJM), involving eligibility screening of titles, abstracts and full papers. Screening questions are listed in online supplemental appendix 2.

\section{Data collection and quality assessment}

The main outcome measure was the diagnostic capability for OCT in detecting $\mathrm{IH}$, expressed as diagnostic accuracy or by appropriate statistical testing.

Secondary outcome measures were:

- Condition(s) associated with IH per study.

- OCT device(s) used.

- OCT success rate.

- Other surrogate estimates of ICP.

- ICP range determined as normal.

Our data extraction tool was adapted from the Cochrane Collaboration (online supplemental appendix 3) ${ }^{29}$ Evidence levels were graded by two independent graders (SRR and $\mathrm{MH}$ ) as per the Oxford $\mathrm{CEBM}^{28}$ and the National Institutes of Health (NIH) Quality Assessment Tool for Observational Studies ${ }^{30}$ was applied for

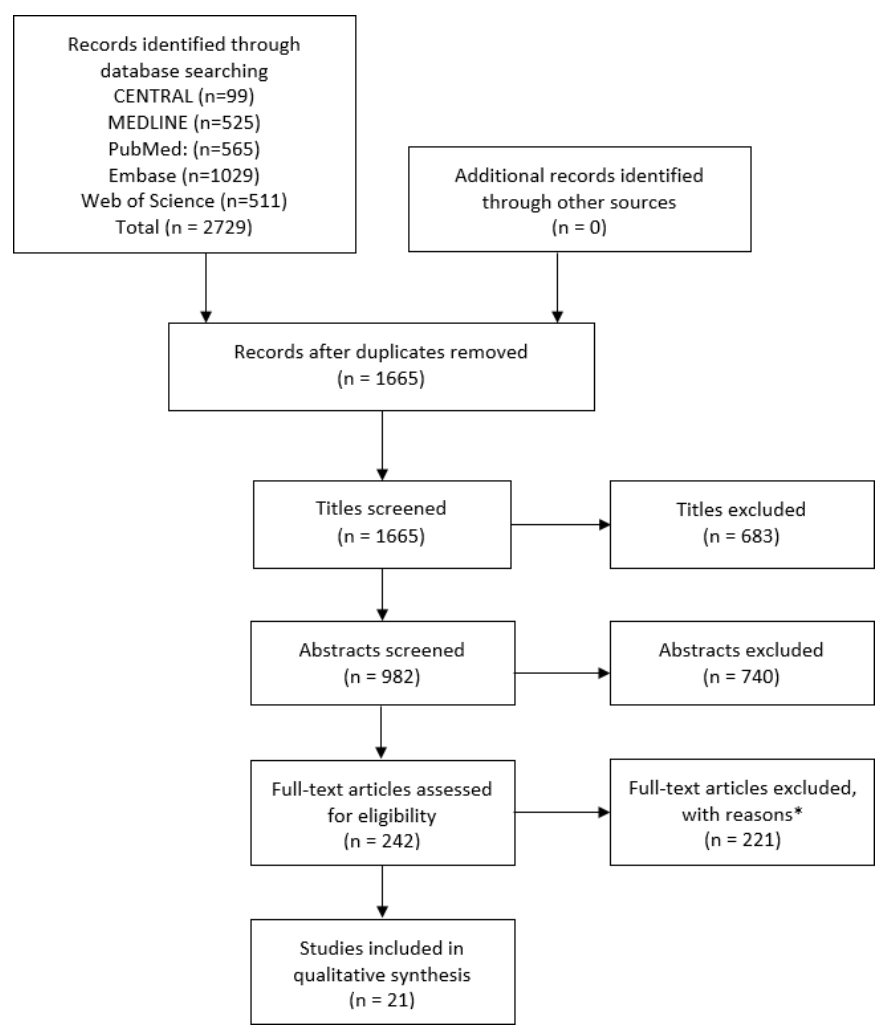

Figure 1 PRISMA study inclusion flow diagram. *Reasons for exclusion were as follows: adult studies $(n=98)$; mixed studies of adults and children without breakdown $(n=27)$; conference abstracts $(n=86)$; case reports $(n=6)$; correspondence $(n=4)$. Online supplemental table 1 contains the full list of excluded studies with reasons. CENTRAL, Cochrane Central Register of Controlled Trials; PRISMA, Preferred Reporting Items for Systematic Reviews and MetaAnalyses.

individual study quality grading (online supplemental appendix 4).

\section{Patient and public involvement}

There was no patient and public involvement specific to this systematic review. However, this group has commenced prospective research using handheld OCT in paediatric IH featuring substantial patient and public involvement, which will be reported separately.

\section{RESULTS}

Our search was executed on 2 April 2020 and data extraction completed on 12 July 2020. Our search returned 2729 records in total, 1665 following deduplication. Following full-text screening, 21 studies were eligible for inclusion in our review (figure 1). Online supplemental table 1 contains the list of excluded articles with reasons. Table 1 summarises the study characteristics and quality assessment of the 21 included studies. One study $^{11}$ was written in Polish and translated by an interpreter, while the remaining 20 were written in English. Nine studies were prospective $\mathrm{e}^{22332-38}$ while 12 were retrospective. ${ }^{3139-49}$ No randomised controlled trials (RCTs) or 
systematic reviews were eligible for inclusion. Following review of the included studies, it was deemed inappropriate to perform quantitative synthesis due to inconsistency in study design and methodology, particularly OCT parameters and reference standards. Therefore, qualitative synthesis was performed.

This review identified studies of craniosynostosis ( $\mathrm{n}=354$ patients), idiopathic IH (IIH; $\mathrm{n}=102)$, spaceoccupying lesion (SOL; $\mathrm{n}=42$ ) and other pathologies $(n=29)$ associated with risk of IH. OCT measures in these studies included optic nerve parameters, rim parameters and retinal parameters. Main outcome measures are displayed in table 2. Secondary outcome measures are reported per condition.

\section{Evidence summary: craniosynostosis}

Craniosynostosis is characterised by the premature, pathological fusion of one or more cranial sutures. This restriction in skull growth can cause IH. Six studies in this review utilised OCT in patients with craniosynostosis. ${ }^{22} 2333344049$ These studies included a total of 393 participants, of which 354 were diagnosed with craniosynostosis. ${ }^{22} 2333344049$ In one comparative study, ${ }^{22} 5$ positive controls had hydrocephalus and suspected $\mathrm{IH},{ }^{22}$ while 34 were normal controls. ${ }^{22}$ OCT devices used include the iVue $^{22}{ }^{23}$ (Optovue, Fremont, California, USA; software V.3.2) and Spectralis ${ }^{33} 344049$ (Heidelberg Engineering, Heidelberg, Germany).

OCT parameters evaluated include retinal nerve fibre layer (RNFL) thickness, ${ }^{22} 2340$ maximal retinal thickness $^{22} 23333449$ and anterior retinal projection. ${ }^{22} 23$ OCT parameters demonstrated good diagnostic capability for IH in craniosynostosis (table 2). Increased RNFL thickness, maximal retinal thickness and anterior retinal projection were associated with papilloedema, while RNFL thinning was associated with optic atrophy. Using single, on-table ICP measures, Swanson et a $t^{22}$ demonstrated good sensitivity (89\%) and specificity $(62 \%)$ of combined RNFL thickness and maximal anterior retinal projection in detecting IH (figure 2). They found that maximal RNFL thickness exceeding $207 \mu \mathrm{m}$ or maximal anterior retinal projection exceeding $159 \mu \mathrm{m}$ in either eye corresponded to the 97.5 th percentile of healthy control patients, thereby representing IH; these figures did not vary significantly based on age. ${ }^{22}$

Reported OCT imaging success rates were high. Driessen $e t a \hat{l}^{34}$ reported overall OCT success in $85 \%$ of eyes. Dagi $e t a t^{40}$ did not specify success rate, but reported that $16.9 \%$ were excluded due to limited cooperation, severe nystagmus, poor scan quality or retinal degeneration. Swanson et al implied $100 \%$ success rate in both studies, ${ }^{22}{ }^{23}$ but acquired OCT images under general anaesthesia and therefore were non-reliant on patient cooperation. Other studies of craniosynostosis did not report OCT success rates.

Apart from OCT, other surrogate measures of ICP displayed poor sensitivity, limiting their potential as screening tools for IH when used in isolation. These included fundoscopy, ${ }^{22} 233340$ visual acuity, ${ }^{40}$ visual fields, ${ }^{40}$ radiological signs 222340 and clinical history including complaints of headache. ${ }^{22} 23$

With respect to defining $\mathrm{IH}$, there is no universally agreed clinical consensus on timing, frequency and duration for accurate ICP measurement, or indeed what figure constitutes raised ICP. ${ }^{50}$ In the three studies using ICP measurements, ${ }^{22} 3349$ ICP $<10 \mathrm{~mm} \mathrm{Hg}$ was determined as normal, while $10-15 \mathrm{~mm} \mathrm{Hg}$ was determined as borderline and $>15 \mathrm{~mm} \mathrm{Hg}$ was determined as raised.

\section{Evidence summary: IIH}

$\mathrm{IIH}$, or primary IH, is characterised by raised ICP in the context of normal CSF composition and no evidence of SOL or ventriculomegaly on neuroimaging. ${ }^{14}$ Principles from the modified Dandy criteria ${ }^{51}$ and the revised criteria by Friedman $e t a \tilde{l}^{22}$ can assist in making diagnosis without ambiguity. Eight studies in this review utilised OCT in patients with IIH. ${ }^{35} 36424345-48$ These studies included a total of 237 participants, of which 102 were diagnosed with IIH. ${ }^{35} 36424345-48$ Of the other included participants in these studies, 74 had pseudopapilloedema, ${ }^{43} 46483$ had SOL, ${ }^{43} 6$ had other pathology ${ }^{43}$ covered below and 52 were normal controls. ${ }^{354} 48$ OCT devices used include the Cirrus HD-OCT ${ }^{14}$ (Carl Zeiss Meditec, Dublin, California, USA), Spectralis ${ }^{42} 48$ and Stratus ${ }^{35} 47$ (Carl Zeiss Meditec). Three studies did not specify which OCT device was used. ${ }^{43} 4546$

OCT parameters included RNFL thickness, ${ }^{35} 36424748$ macular volume,$^{35}$ disruption of the ellipsoid zone ${ }^{42}$ and Bruch's membrane opening. ${ }^{48}$ Two studies did not specify which OCT parameters were used. ${ }^{45} 46$ OCT demonstrated good diagnostic capability in IIH (table 2). Increased RNFL thickness 35364348 and macular volume $^{35}$ were associated with IH, while RNFL thinning and disruption of the ellipsoid zone were associated with optic atrophy and vision loss. ${ }^{42}$ In addition, Thompson et $a l^{48}$ found that the transverse diameter of Bruch's membrane opening was enlarged in mild papilloedema and could be used together with RNFL thickness to distinguish mild papilloedema from psuedopapilloedema (figure 3).

Lee $e t a l^{36}$ reported $86.7 \%$ OCT imaging success rate, while Sánchez-Tocino et al ${ }^{47}$ reported $100 \%$ OCT success. Other studies of IIH did not report success rates.

With regard to other surrogate measures of ICP, Ozturk $e t a l^{46}$ found that optic nerve sheath diameter was moderately associated with CSF opening pressure $(\mathrm{r}=0.661 ; \mathrm{p}<0.005)$. Lee $e t a l^{36}$ found that body mass index was moderately associated with lumbar puncture opening pressure $(\mathrm{r}=0.607 ; \mathrm{p}=0.028)$. Headache characteristics did not reliably detect children with IIH. $^{35} 36$ 45-47

With respect to ICP measurements, only Krishnakumar et $a t^{45}$ provided definitions for normal and raised ICP, using lumbar CSF opening and steady state pressures: $<15$ $\mathrm{mm} \mathrm{Hg}=$ normal $\mathrm{CSF}$ pressure; $>20 \mathrm{~mm} \mathrm{Hg}=$ high pressure. 


\section{A. Retinal thickness}

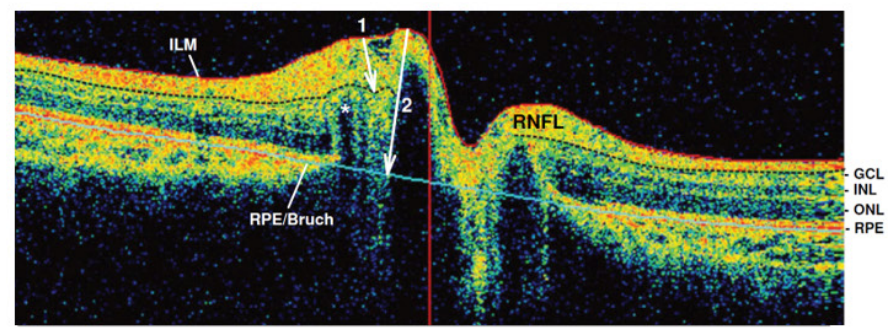

\section{B. Anterior retinal projection}

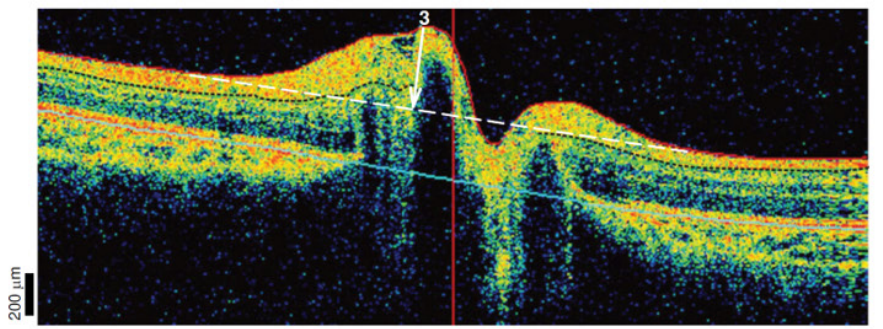

C. Normal and elevated ICP
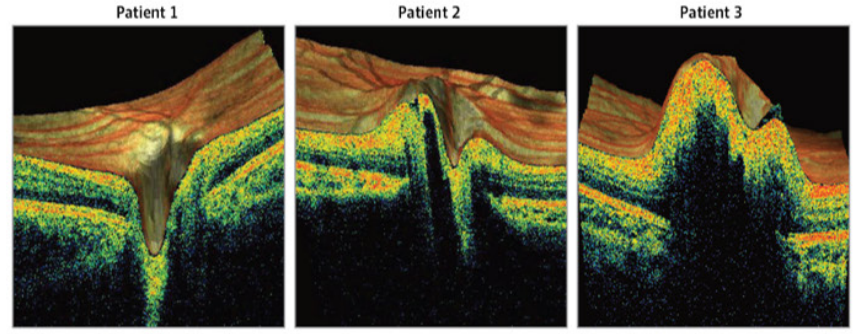

\section{OCT retinal parameters $\begin{aligned} & \text { OCraniosynostosis } \\ & \text { OPositive Controls }\end{aligned}$}
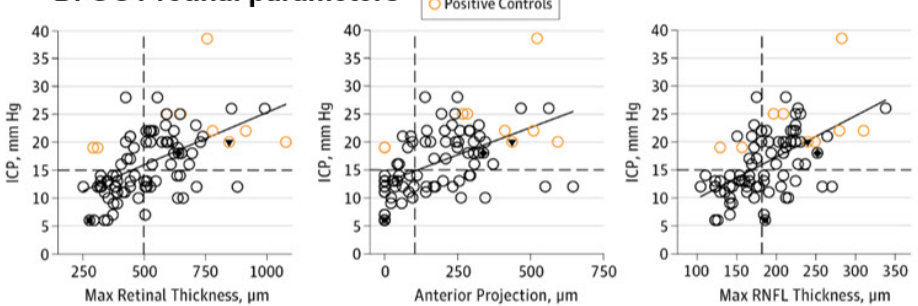

\section{E. ROC curves}
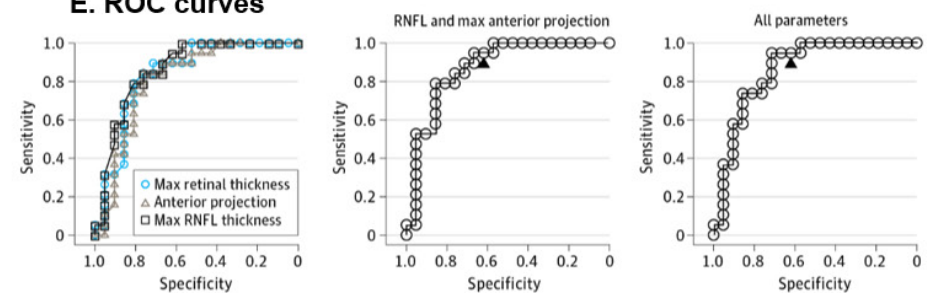

Figure 2 Optical coherence tomographic (OCT) parameters, variation with intracranial pressure (ICP) and utility as a screening test. (A) 1. RNFL thickness; 2. Retinal thickness; *indicates vascular elements causing posterior shadowing; (B) 3. Anterior retinal projection, where the dotted white line is a vector connecting the posterior-most ILM adjacent to either side of the optic disc. (C) OCT images of patients with normal (patient 1; ICP, $6 \mathrm{~mm} \mathrm{Hg}$ ) and elevated (patient 2; ICP, $18 \mathrm{~mm} \mathrm{Hg}$ and patient 3; ICP, $20 \mathrm{~mm}$ Hg) ICP. (D) OCT retinal parameters (maximal retinal thickness, anterior projection and maximal RNFL thickness) plotted as a function of ICP measured intraoperatively. (E) ROC curves for each of the three OCT parameters, combined RNFL and maximal retinal thickness parameters, and a model combining all parameters. GCL, ganglion cell layer; ILM, inner limiting membrane; INL, inner nuclear layer; ONL, outer nuclear layer; RNFL, retinal nerve fibre layer; ROC, receiver operating characteristic; RPE, retinal pigment epithelium. Reprinted with permission from: Swanson et al. ${ }^{22}$ Copyright @ 2017 , American Medical Association.

\section{Evidence summary: SOL}

Intracranial SOL include tumours or abscesses within the cranial cavity, which are associated with IH. Five studies in this review used OCT in patients with SOL. ${ }^{37} 39414344$ These studies included a total of 121 participants, of which 42 had SOL. ${ }^{3739414344}$ Of the remaining participants, 2 

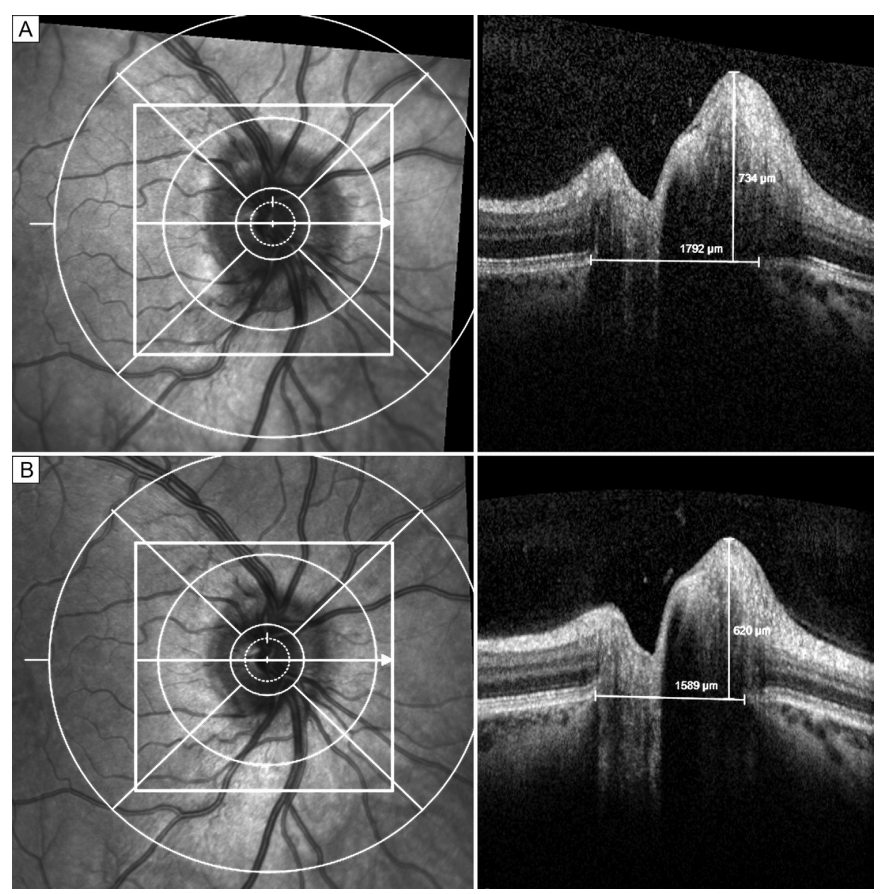

Figure 3 Measuring the transverse horizontal diameter of Bruch's membrane opening (BMO) and the papillary height on SD-OCT. (A) When the ONH is swollen from mild papilloedema; $(\mathrm{B})$ when the papilloedema has resolved. $\mathrm{ONH}$, optic nerve head; SD-OCT, spectral domain optical coherence tomography. Reprinted with permission from: Thompson et al. ${ }^{48}$ Copyright (C 2017, Elsevier.

had IIH, ${ }^{43} 9$ had psuedopapilloedema, ${ }^{43} 10$ had other pathology ${ }^{37} 41$ covered below and 58 had optic disc drusen with no intracranial pathology. ${ }^{41}$ The following OCT devices were used: Cirrus HD-OCT, ${ }^{39} 44$ DRI-OCT-1 Atlantis, ${ }^{37}$ Spectralis ${ }^{41}$ and Stratus. ${ }^{39}$ Malem et $a t^{43}$ did not specify which device was used.

OCT parameters included RNFL thickness, ${ }^{39} 414344$ ganglion cell layer thickness, ${ }^{44}$ anterior bowing of Bruch's membrane, ${ }^{41}$ neural canal diameter, ${ }^{37}$ papillary vertical height $^{37}$ and anterior lamina cribrosa depth. ${ }^{37}$ OCT demonstrated value as part of the clinical workup of these children (table 2). RNFL thinning was associated with optic atrophy ${ }^{39}$ and visual field loss, ${ }^{39} 44$ as was ganglion cell loss. ${ }^{44}$ Lee et $a l^{37}$ highlighted the reversibility of papilloedema in patients with SOL following ICP reducing surgery, whereby mean neural canal diameter and papillary vertical height decreased while mean anterior lamina cribrosa depth increased (figure 4). Dahlmann-Noor et $a l^{41}$ reported one patient with intraventricular tumour with increased temporal RNFL thickness, but no bowing of Bruch's membrane.

Dahlmann-Noor et $a l^{41}$ reported OCT imaging success rate of $100 \%$, while other SOL studies did not report success rate.

Clinical history including headache characteristics did not reliably detect children with SOL. ${ }^{39} 41$ Humphrey visual field testing is not designed for children and was difficult or not feasible in many cases. ${ }^{39} 44$

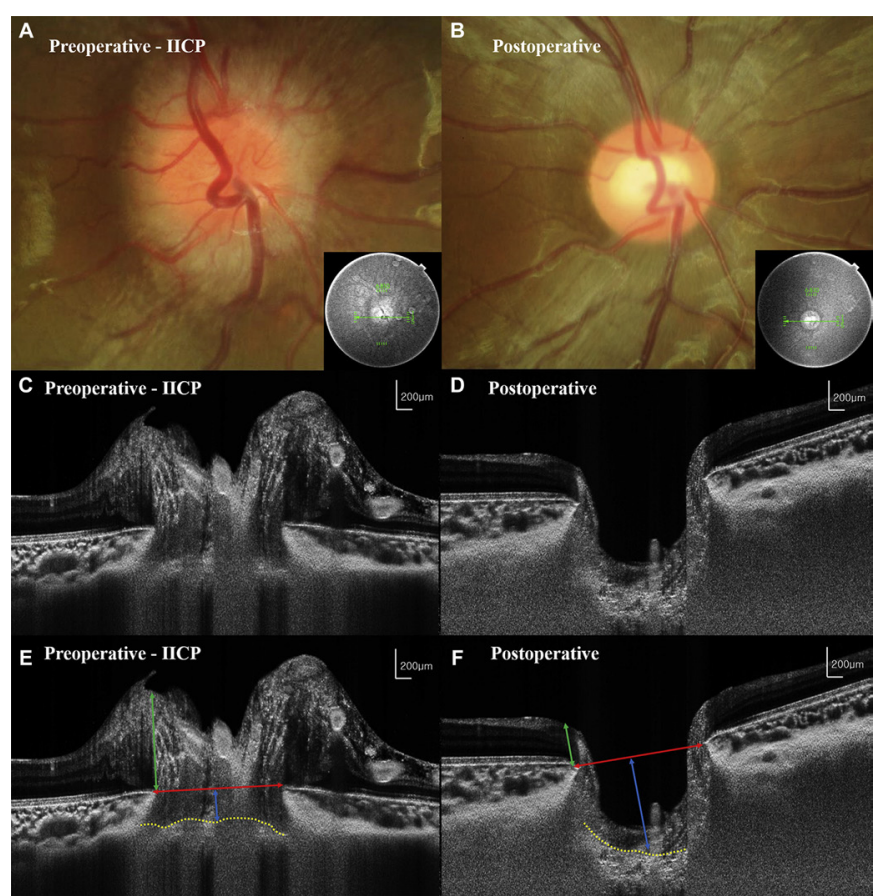

Figure 4 Swept-source optical coherence tomography (SS-OCT) images (horizontal scan) of optic nerve head structures in the right eye of an 8-year-old boy with pilocytic astrocytoma. Disc photographs showing preoperative papilloedema (A) and postoperative resolution of the same (B) are presented with the indicating OCT section (small squares). A row SS-OCT image of preoperative (C) and postoperative (D) states can be observed. Measurements are obtained using the preoperative $(\mathrm{E})$ and postoperative (F) images. After surgical decompression, the neural canal diameter (red line) and papillary vertical height (green line) have decreased and the lamina cribrosa (LC) shows posterior displacement (yellow dotted line; anterior surface of the LC contour). IICP, increased intracranial pressure. Reprinted with permission from: Lee et al. ${ }^{37}$ Copyright (C 2017, Elsevier.

Lee $e t a l^{37}$ reported mean preoperative and postoperative ICP values of $24.0 \pm 5.0$ and $13.2 \pm 6.3 \mathrm{~mm} \mathrm{Hg}$, but no SOL study defined a normal range for ICP. ${ }^{3941}$

\section{Evidence summary: other pathology}

Seven studies 22313237384143 included children with other pathology associated with IH, including hydrocephalus $^{22} 3738 \quad(n=16)$, papilloedema of unspecified aetiology $^{32}(n=5)$, acute lymphoblastic leukaemia ${ }^{41}(n=1)$, aseptic meningitis $^{43}(n=1)$, growth hormone replacement therapy $^{31}(n=1)$, head injury ${ }^{43}(n=1)$, recurrent nephrotic syndrome $^{31}(n=1)$, rickets ${ }^{41}(n=1)$, sagittal sinus throm$\operatorname{bosis}^{43}(n=3)$ and sigmoid sinus thrombosis ${ }^{43}(n=1)$. Of note, Tran-Viet et $a l^{38}$ used the Envisu handheld OCT system (C2200 and C2300, Bioptigen, Research Triangle Park, North Carolina, USA), with which they successfully scanned 25 out of $26(96 \%)$ undilated eyes of conscious infants without sedation. Other OCT devices used were the Spectralis and DRI-OCT-1 Atlantis; Malem et $a t^{43}$ did not specify which device was used.

OCT parameters used in these studies included RNFL thickness, ${ }^{31} 324143$ volumetric spectral domain 
(SD) OCT of optic nerve head, ${ }^{32}$ volumetric enhanced depth imaging (EDI) OCT of optic nerve head, ${ }^{32}$ neural canal diameter, ${ }^{37}$ papillary vertical height, ${ }^{37}$ anterior lamina cribrosa depth ${ }^{37}$ and anterior bowing of Bruch's membrane $^{41}$ (table 2). Tran-Viet $e t a l^{38}$ did not specify which OCT parameters were used. Chang $e t a \vec{l}^{2}$ found a positive association between optic nerve head volume on SD-OCT and EDI-OCT with papilloedema, but did not specify aetiology. Other associations between OCT parameters and IH have been described above.

OCT imaging success rates were high in these studies: Tran-Viet $e t a l^{8}$ reported $96 \%$ success, Chang et $a l^{2}$ reported $98 \%$ and Dahlmann-Noor et $a l^{41}$ reported $100 \%$. Mrugacz $e t a l^{31}$ and Swanson et $a l^{2223}$ implied $100 \%$ success rates. Other studies did not report OCT success rates.

\section{DISCUSSION}

This systematic review collated a body of evidence evaluating the role of OCT to detect IH, specifically the structural changes associated with papilloedema and optic atrophy. This review could not recommend the widespread use of OCT in all children at risk of IH as standard clinical practice, as no level 1 studies were identified for these conditions. However, this review recognises the value of OCT in paediatric $\mathrm{IH}$, particularly in cases where ICP status is uncertain or borderline, for a number of reasons: (1) OCT enables rapid, non-invasive, quantitative cross-sectional measurements of the optic nerve head and retina, not offered by conventional funduscopy; (2) OCT permits serial measurements to help appreciate evolution in optic nerve head and retinal changes over time, where applicable and (3) OCT could guide and support the overall clinical workup of affected children. Further prospective validating studies are required to develop formal clinical guidelines for OCT in this role.

\section{Quality of evidence}

Two independent graders (SRR and MH) identified varying levels of evidence among the 21 included studies, as per the Oxford CEBM. ${ }^{28}$ There were no systematic reviews, RCTs or validating cohort studies measuring OCT parameters against established reference standards, hence no study was graded as level 1 evidence. The NIH Quality Assessment Tool for individual studies identified 10 studies of fair quality and 11 studies of poor quality (table 1). The major limiting factor to quality was use of other surrogate clinical measures as the reference standard for IH, as opposed to direct intraparenchymal ICP monitoring.

\section{Research in context}

There is a paucity of clinical guidelines for the management of paediatric IH in general. Searches for relevant clinical guidelines were performed via PubMed using the search terms "guidelines" AND "optical coherence tomography" AND "idiopathic intracranial hypertension" OR "craniosynostosis" OR "space occupying lesion", which returned two relevant guidelines. Mollan et $a \tilde{l}^{\tilde{3}}$ recently published the first consensus guidelines on management of IIH, which state: 'Where visual function is found to be threatened, regular ophthalmic examination must occur because this will influence timely management... Formal documentation of the optic nerve head appearance, such as serial photographs or OCT imaging, is useful.' This is consistent with our review's findings. The guidelines by Mollan et $a l^{53}$ apply to all patients with IIH and are not specific to children. The Working Group on Craniosynostosis $^{54}$ published clinical guidelines for craniosynostosis in 2015, but these did not feature OCT. No relevant guidelines were identified for the use of OCT in SOL. The same search strategy was used for nice.org.uk-the UK's National Institute for Health and Care Excellence, which returned no relevant guidelines.

The NORDIC Idiopathic Intracranial Hypertension Treatment Trial is a landmark RCT with a dedicated OCT sub-study committee. ${ }^{55}$ Although the resulting papers appeared in our systematic search, they were excluded from our review because they excluded children aged under 18. On further reading, positive associations between ICP and RNFL thickness, total retinal thickness and optic nerve head volume were also found, consistent with our review findings. ${ }^{55}$

This review identified studies using a range of OCT devices. Conventional, table-mounted OCT devices such as the Spectralis may be suitable in school-age children, but are not designed for young infants. While high OCT imaging success rates were reported, many studies were limited to school aged children rather than young infants. By contrast, this review found that the portable iVue device was successfully used for on-table OCTs in young infants under general anaesthesia, ${ }^{22} 23$ while the Envisu handheld OCT was used in conscious newborns without the need for general anaesthesia or pupil dilation in one feasibility study. ${ }^{38}$

Of note, handheld OCT has been recently used to describe the normal development of the optic nerve ${ }^{27}$ and fovea ${ }^{15} 16$ in infants and children, with excellent feasibility. Handheld OCT has also been utilised in a wide range of other pedatric conditions including retinopathy of prematurity ${ }^{56}$ nystagmus, ${ }^{57}$ albinism, ${ }^{58}$ achromatopsia, ${ }^{59}$ foveal hypoplasia, ${ }^{60}$ optic nerve hypoplasia, ${ }^{61}$ primary congenital glaucoma ${ }^{62}$ microcephaly ${ }^{63}$ and others. Handheld OCT may be better tolerated in young children, particularly those with craniosynostosis associated with cognitive delay. However, further research is required to validate this. Therefore, our group has recently commenced prospective research using handheld OCT in craniosynostosis. ${ }^{64}$

\section{Strengths and limitations}

This systematic review has a number of strengths. To the best of our knowledge, this is the first systematic review assessing the role of OCT in paediatric IH. Indeed, no such other systematic review appeared in our systematic search. PRISMA guidelines ${ }^{24}$ and rigorous Cochrane 
methods were followed. ${ }^{25}$ Our protocol was registered on PROSPERO ${ }^{26}$ and published ${ }^{27}$ prior to this study, to promote transparency and avoid duplication. Two independent screeners (SRR and RJM) conducted the systematic search and two independent graders (SRR and MH) completed the quality assessment. A broad search strategy was developed with support from an experienced research librarian. Notably, our search terms did not restrict to certain conditions or age-related keywords-rather, the records were manually searched to avoid missing any key evidence. There were no time or language restrictions, yielding a broad range of eligible studies for inclusion including one article translated from Polish. ${ }^{31}$

We also acknowledge the limitations of this review. Meta-analysis was not possible due to the absence of eligible RCTs and inconsistency in OCT measures and reference standards. The maximum grade of recommendations from this review was grade B, based on consistent level 2 or 3 studies or extrapolations from level 1 studies ${ }^{28}$ Notably, while some studies demonstrated OCT detection of papilloedema (eg, RNFL thickening), other studies demonstrated OCT detection of optic atrophy (eg, RNFL thinning), therefore, caution must be exercised in interpreting OCT changes within the full clinical context and in serial examinations as far as possible. No level 1 evidence was returned by this review, which would be required to identify optimal OCT parameters and develop formal clinical guidelines. The broad search strategy resulted in a large number of records to screen, although this reduced the risk of missing key evidence.

\section{Further research}

This review highlighted the lack of standardisation in OCT parameters used to detect IH in children. Further research is required to clarify the most appropriate OCT parameters for this purpose, using gold-standard ICP measures. This could be achieved by a validating prospective study using existing OCT reference standards. This should qualify as level $1 \mathrm{~b}$ evidence as per the Oxford CEBM. ${ }^{28}$ Handheld OCT could enable serial imaging in young infants without sedation, which would be particularly valuable as many pathologies associated with paediatric ICP can begin from birth, however current evidence is lacking. Serial OCT imaging could enable appreciation of evolving optic nerve head and retinal changes over time, where applicable. Further high quality prospective research could help to integrate OCT into formal clinical guidelines and clinical decision-making algorithms.

Other interesting research questions that were not answered in this systematic review include the following. First, how should OCT parameters be interpreted following IH where optic atrophy has occurred? The full clinical context, including visual function, may help indicate whether the patient has optic atrophy, rather than drawing conclusions based on OCT findings in isolation. Second, how should OCT parameters be interpreted in patients with gliosis of the optic nerve in chronic IH? This could prevent the typical ONH changes expected on
OCT. Third, how should OCT be interpreted in recurrent $\mathrm{IH}$ which can occur in patients with ventriculoperitoneal or lumbar-peritoneal shunt obstruction? Again, gliosis may prevent the typical ONH changes expected on OCT. It is likely that OCT must be interpreted in the full clinical context, including visual function, to optimise diagnosis and management of these complex cases.

\section{CONCLUSIONS}

This systematic review has recognised the diagnostic potential of OCT in paediatric IH in craniosynostosis, IIH, SOL and other pathology, in conjunction with established clinical measures of ICP, to guide diagnosis and management. However, this review could not recommend the development of formal guidelines, nor the widespread use of OCT in all children at risk of IH as standard clinical practice at this stage. Further validating prospective research is required to improve our understanding of the clinical utility of OCT in this role (including handheld OCT), to establish optimal OCT parameters for paediatric IH and to inform formal clinical guidelines.

\section{Twitter Sohaib R Rufai @0phthoReg}

Acknowledgements We thank the authors of the included studies for providing further data where required for our systematic review. We thank Dr Jordan Swanson, MD, for assisting with the adaptation of Figure 2 for this systematic review. We thank Selina Lock, Research Services Consultant at the University of Leicester David Wilson Library, for providing expert guidance in our systematic search strategy. We thank Krystyna Ulanicka for providing the English translation of the included Polish study.

Contributors Authorship and contribution: SRR: Design and conceptualisation of the study; major role in the acquisition of data; analysis and interpretation of the data; drafted the manuscript for intellectual content. $\mathrm{MH}$ : Analysis and interpretation of the data; revised the manuscript for intellectual content. NuOJ: Design and conceptualisation of the study; revised the manuscript for intellectual content. RJM: Guarantor; design and conceptualisation of the study; major role in the acquisition of data; analysis and interpretation of the data; revised the manuscript for intellectual content.

Funding SRR is funded by a National Institute for Health Research (NIHR) Doctoral Fellowship (Award ID: NIHR300155) for this research project. This publication presents independent research funded by the MRC and National Institute for Health Research (NIHR).

Disclaimer The views expressed are those of the author(s) and not necessarily those of the MRC, the NHS, the NIHR or the Department of Health and Social Care.

Competing interests None declared.

Patient consent for publication Not required.

Ethics approval No ethical approval was required to conduct this systematic review.

Provenance and peer review Not commissioned; externally peer reviewed.

Data availability statement No data are available.

Supplemental material This content has been supplied by the author(s). It has not been vetted by BMJ Publishing Group Limited (BMJ) and may not have been peer-reviewed. Any opinions or recommendations discussed are solely those of the author(s) and are not endorsed by BMJ. BMJ disclaims all liability and responsibility arising from any reliance placed on the content. Where the content includes any translated material, BMJ does not warrant the accuracy and reliability of the translations (including but not limited to local regulations, clinical guidelines, terminology, drug names and drug dosages), and is not responsible for any error and/or omissions arising from translation and adaptation or otherwise.

Open access This is an open access article distributed in accordance with the Creative Commons Attribution 4.0 Unported (CC BY 4.0) license, which permits 
others to copy, redistribute, remix, transform and build upon this work for any purpose, provided the original work is properly cited, a link to the licence is given, and indication of whether changes were made. See: https://creativecommons.org/ licenses/by/4.0/.

ORCID iD

Sohaib R Rufai http://orcid.org/0000-0001-8134-6393

\section{REFERENCES}

1 Quincke $\mathrm{H}$. Ueber meningitis serosa und verwandte Zustände. Deutsche Zeitschrift für Nervenheilkunde 1896;9:149-68.

2 Matthews Y-Y, Dean F, Lim MJ, et al. Pseudotumor cerebri syndrome in childhood: incidence, clinical profile and risk factors in a national prospective population-based cohort study. Arch Dis Child 2017;102:715-21.

3 Gillson N, Jones C, Reem RE, et al. Incidence and demographics of pediatric intracranial hypertension. Pediatr Neurol 2017;73:42-7.

4 Derderian C, Seaward J. Syndromic craniosynostosis. Semin Plast Surg 2012;26:064-75.

5 Jordan CO, Aylward SC. Intracranial hypertension: a current review. Curr Opin Pediatr 2018;30:764-74.

6 Zhang X, Medow JE, Iskandar BJ, et al. Invasive and noninvasive means of measuring intracranial pressure: a review. Physiol Meas 2017;38:R143-82.

7 Tamburrini G, Caldarelli M, Massimi L, et al. Intracranial pressure monitoring in children with single suture and complex craniosynostosis: a review. Childs Nerv Syst 2005;21:913-21.

8 Tuite GF, Chong WK, Evanson J, et al. The effectiveness of papilledema as an indicator of raised intracranial pressure in children with craniosynostosis. Neurosurgery 1996;38:272-8.

9 Dubourg J, Javouhey E, Geeraerts T, et al. Ultrasonography of optic nerve sheath diameter for detection of raised intracranial pressure: a systematic review and meta-analysis. Intensive Care Med 2011;37:1059-68.

10 Driessen C, Bannink N, Lequin M, et al. Are ultrasonography measurements of optic nerve sheath diameter an alternative to funduscopy in children with syndromic craniosynostosis? J Neurosurg Pediatr 2011;8:329-34.

11 Tuite GF, Evanson J, Chong WK, et al. The beaten copper cranium: a correlation between intracranial pressure, cranial radiographs, and computed tomographic scans in children with craniosynostosis. Neurosurgery 1996;39:691-8.

12 Thompson DA, Liasis A, Hardy S, et al. Prevalence of abnormal pattern reversal visual evoked potentials in craniosynostosis. Plast Reconstr Surg 2006;118:184-92.

13 Andersson L, Sjölund J, Nilsson J. Flash visual evoked potentials are unreliable as markers of ICP due to high variability in normal subjects. Acta Neurochir 2012;154:121-7.

14 Lee H, Proudlock FA, Gottlob I. Pediatric optical coherence tomography in clinical Practice-Recent progress. Invest Ophthalmol Vis Sci 2016;57:OCT69-79.

15 Patel A, Purohit R, Lee H, et al. Optic nerve head development in healthy infants and children using handheld spectral-domain optical coherence tomography. Ophthalmology 2016;123:2147-57.

16 Lee $\mathrm{H}$, Purohit R, Patel A, et al. In vivo foveal development using optical coherence tomography. Invest Ophthalmol Vis Sci 2015;56:4537-45.

17 Optical coherence tomography substudy Committee; Nordic idiopathic intracranial hypertension Study Group. papilledema outcomes from the optical coherence tomography substudy of the idiopathic intracranial hypertension treatment trial. Ophthalmology 2015;122:1939-45.

18 Scott CJ, Kardon RH, Lee AG, et al. Diagnosis and grading of papilledema in patients with raised intracranial pressure using optical coherence tomography vs clinical expert assessment using a clinical staging scale. Arch Ophthalmol 2010;128:705-11.

19 Skau M, Yri H, Sander B, et al. Diagnostic value of optical coherence tomography for intracranial pressure in idiopathic intracranial hypertension. Graefes Arch Clin Exp Ophthalmol 2013;251:567-74.

20 Anand A, Pass A, Urfy MZ, et al. Optical coherence tomography of the optic nerve head detects acute changes in intracranial pressure. $J$ Clin Neurosci 2016;29:73-6.

21 Patel MD, Malhotra K, Shirazi Z, et al. Methods for quantifying optic disc volume and peripapillary deflection volume using radial optical coherence tomography scans and association with intracranial pressure. Front Neurol 2019;10:798.

22 Swanson JW, Aleman TS, Xu W, et al. Evaluation of optical coherence tomography to detect elevated intracranial pressure in children. JAMA Ophthalmol 2017;135:320-8.
23 Swanson JW, Xu W, Ying G-S, et al. Intracranial pressure patterns in children with craniosynostosis utilizing optical coherence tomography. Childs Nerv Syst 2020;36:535-44.

24 Moher D, Liberati A, Tetzlaff J, et al. Preferred reporting items for systematic reviews and meta-analyses: the PRISMA statement. Ann Intern Med 2009;151:264-W64.

25 Training C. Cochrane Handbook for Systematic Reviews of Interventions, 2019. Available: https://training.cochrane.org/ handbook [Accessed 10 Aug 2020].

26 Rufai S. Detection of intracranial hypertension in children using optical coherence tomography: a systematic review. Prospero 2019 CRD42019154254. Available: https://www.crd.york.ac.uk/prospero/ display_record.php?ID=CRD42019154254 [Accessed 10 Aug 2020].

27 Rufai SR, Jeelani NUO, McLean RJ. Detection of intracranial hypertension in children using optical coherence tomography: a systematic review protocol. BMJ Open 2020;10:e037833.

28 Oxford Centre for Evidence-based Medicine. Levels of evidence, 2009. Available: https://www.cebm.net/2009/06/oxford-centreevidence-based-medicine-levels-evidence-march-2009/ [Accessed 10 Aug 2020].

29 Cochrane. data extraction forms, 2019. Available: https://dplp. cochrane.org/data-extraction-forms [Accessed 10 Aug 2020].

$30 \mathrm{NIH}$. Study quality assessment tools: quality assessment tool for observational cohort and cross-sectional studies. Available: https:// www.nhlbi.nih.gov/health-topics/study-quality-assessment-tools [Accessed 10 Aug 2020].

31 Mrugacz M, Szumiński M, Bakunowicz-Łazarczyk A. Optyczna koherentna tomografia w diagnostyce nadciśnienia wewnatrzczaszkowego u dzieci i młodziezy [Optical coherence tomography in diagnosis of intracranial hypertension in children]. Klin Oczna 2011;113:258-62.

32 Chang MY, Velez FG, Demer JL, et al. Accuracy of diagnostic imaging modalities for classifying pediatric eyes as papilledema versus pseudopapilledema. Ophthalmology 2017;124:1839-48.

33 den Ottelander BK, de Goederen R, van Veelen MC. Muenke syndrome: long-term outcome of a syndrome-specific treatment protocol [published online ahead of print, 2019 Jul 19]. J Neurosurg Pediatr 2019:1-8.

34 Driessen C, Eveleens J, Bleyen I, et al. Optical coherence tomography: a quantitative tool to screen for papilledema in craniosynostosis. Childs Nerv Syst 2014;30:1067-73.

35 El-Dairi MA, Holgado S, O'Donnell T, et al. Optical coherence tomography as a tool for monitoring pediatric pseudotumor cerebri. $J$ Aapos 2007;11:564-70.

36 Lee YA, Tomsak RL, Sadikovic Z, et al. Use of ocular coherence tomography in children with idiopathic intracranial Hypertension-A single-center experience. Pediatr Neurol 2016;58:101-6.

37 Lee WJ, Kim HJ, Park KH, et al. Change in optic nerve after intracranial pressure reduction in children. Ophthalmology 2017;124:1713-5.

38 Tran-Viet D, Wong BM, Mangalesh S, et al. Handheld spectral domain optical coherence tomography imaging through the UNDILATED pupil in infants born preterm or with hypoxic injury or hydrocephalus. Retina 2018;38:1588-94.

39 Bialer OY, Goldenberg-Cohen N, Toledano H, et al. Retinal NFL thinning on OCT correlates with visual field loss in pediatric craniopharyngioma. Can J Ophthalmol 2013;48:494-9.

40 Dagi LR, Tiedemann LM, Heidary G, et al. Using spectral-domain optical coherence tomography to detect optic neuropathy in patients with craniosynostosis. J Aapos 2014;18:543-9.

41 Dahlmann-Noor AH, Adams GW, Daniel MC, et al. Detecting optic nerve head swelling on ultrasound and optical coherence tomography in children and young people: an observational study. $\mathrm{Br}$ J Ophthalmol 2018;102:318-22.

42 Gospe SM, Bhatti MT, El-Dairi MA. Anatomic and visual function outcomes in paediatric idiopathic intracranial hypertension. $\mathrm{Br} J$ Ophthalmol 2016;100:505-9.

43 Malem A, De Salvo G, West S. Use of multicolor imaging in the assessment of suspected papilledema in 20 consecutive children. $J$ Aapos 2016;20:532-6.

44 Mediero S, Noval S, Bravo-Ljubetic L, et al. Visual outcomes, visual fields, and optical coherence tomography in paediatric craniopharyngioma. Neuroophthalmology 2015;39:132-9.

45 Krishnakumar D, Pickard JD, Czosnyka Z, et al. Idiopathic intracranial hypertension in childhood: pitfalls in diagnosis. Dev Med Child Neurol 2014;56:749-55.

46 Ozturk Z, Atalay T, Arhan E, et al. The efficacy of orbital ultrasonography and magnetic resonance imaging findings with direct measurement of intracranial pressure in distinguishing papilledema from pseudopapilledema. Childs Nerv Syst 2017;33:1501-7. 
47 Sánchez-Tocino H, Bringas R, Iglesias D. Utilidad del tomógrafo de coherencia óptica (OCT) en el seguimiento de la hipertensión intracraneal idiopática en la infancia [Utility of optic coherence tomography (OCT) in the follow-up of idiopathic intracranial hypertension in childhood]. Arch Soc Esp Oftalmol 2006;81:383-9.

48 Thompson AC, Bhatti MT, El-Dairi MA. Bruch's membrane opening on optical coherence tomography in pediatric papilledema and pseudopapilledema. J Aapos 2018;22:38-43.

49 van de Beeten SDC, Cornelissen MJ, van Seeters RM. Papilledema in unicoronal Synostosis: a rare finding. J Neurosurg Pediatr 2019:1-6.

50 Hayward R, Britto J, Dunaway D, et al. Connecting raised intracranial pressure and cognitive delay in craniosynostosis: many assumptions, little evidence. J Neurosurg Pediatr 2016;18:242-50.

51 Dandy WE. Intracranial pressure without brain tumor: diagnosis and treatment. Ann Surg 1937;106:492-513.

52 Friedman DI, Liu GT, Digre KB. Revised diagnostic criteria for the pseudotumor cerebri syndrome in adults and children. Neurology 2013;81:1159-65.

53 Mollan SP, Davies B, Silver NC, et al. Idiopathic intracranial hypertension: consensus guidelines on management. $J$ Neurol Neurosurg Psychiatry 2018;89:1088-100.

54 Mathijssen IMJ. Guideline for care of patients with the diagnoses of craniosynostosis: Working group on craniosynostosis. J Craniofac Surg 2015;26:1735-807.

55 , Optical Coherence Tomography Substudy Committee, NORDIC Idiopathic Intracranial Hypertension Study Group. Papilledema outcomes from the optical coherence tomography substudy of the idiopathic intracranial hypertension treatment trial. Ophthalmology 2015;122:1939-45.
56 Anwar S, Nath M, Patel A, et al. Potential utility of foveal morphology in preterm infants measured using hand-held optical coherence tomography in retinopathy of prematurity screening. Retina 2020;40:1592-602.

57 Lee H, Proudlock F, Gottlob I. Is handheld optical coherence tomography reliable in infants and young children with and without nystagmus? Invest Ophthalmol Vis Sci 2013;54:8152-9.

58 Mohammad S, Gottlob I, Sheth V, et al. Characterization of abnormal optic nerve head morphology in albinism using optical coherence tomography. Invest Ophthalmol Vis Sci 2015;56:4611-8.

59 Thomas MG, Kumar A, Kohl S, et al. High-Resolution in vivo imaging in achromatopsia. Ophthalmology 2011;118:882-7.

60 Rufai SR, Thomas MG, Purohit R, et al. Can structural grading of foveal hypoplasia predict future vision in infantile nystagmus?: a longitudinal study. Ophthalmology 2020;127:492-500.

61 Pilat A, Sibley D, McLean RJ, et al. High-Resolution imaging of the optic nerve and retina in optic nerve hypoplasia. Ophthalmology 2015;122:1330-9.

62 Pilat AV, Proudlock FA, Shah S, et al. Assessment of the anterior segment of patients with primary congenital glaucoma using handheld optical coherence tomography. Eye 2019;33:1232-9.

63 Papageorgiou E, Pilat A, Proudlock F, et al. Retinal and optic nerve changes in microcephaly: an optical coherence tomography study. Neurology 2018;91:e571-85.

64 Rufai SR, Bowman R, Bunce C, et al. Feasibility and repeatability of handheld optical coherence tomography in children with craniosynostosis. Trans/ Vis Sci Technol 2021;10:24. 